\title{
Recent DØ results in Diffractive and Jet Physics
}

\author{
Vivian O'Dell ${ }^{\star} \dagger$ \\ Fermi National Accelerator Laboratory, P.O. Box 500, Batavia, IL 60510 \\ E-mail: odell@fnal.gov
}

$\mathrm{D} \emptyset$ measurements of the inclusive jet cross section and the isolated photon cross section are presented along with comparisons to theoretical calculations. A summary of the status of the Forward Proton Detector, used in the study of diffractive and elastic events, is presented along with the analysis status.

International Workshop on Diffraction in High-Energy Physics-Diffraction 06-

Adamantas, Milos island, Greece

5-10 September 2006

\footnotetext{
* Speaker

${ }^{\dagger}$ Presented on behalf of the DØ collaboration
} 


\section{Introduction}

The production rates of particle jets with large transverse momentum $\left(\mathrm{p}_{\mathrm{T}}\right)$ provide sensitive tests for the predictions of perturbative QCD (pQCD) and give information on the nonperturbative structure of the proton as parameterized in the parton density functions (PDFs). In Run II of the Fermilab Tevatron, the center of mass energy was increased from $\sqrt{s}=1.8 \mathrm{TeV}$ to $\sqrt{s}=1.96 \mathrm{TeV}$ resulting in an increased jet cross section at high $\boldsymbol{p}_{T}$. Figure $1 \mathrm{~b}$ ) shows the NLO QCD prediction for the two center of mass energies. In Figure 1a) the reach as a function of $\boldsymbol{x}$ and $Q^{2}$ is shown for many different experiments. The Fermilab Tevatron offers a complementary measurement to H1 or Zeus and probes higher $\boldsymbol{Q}^{2}$ at large $\boldsymbol{x}$.
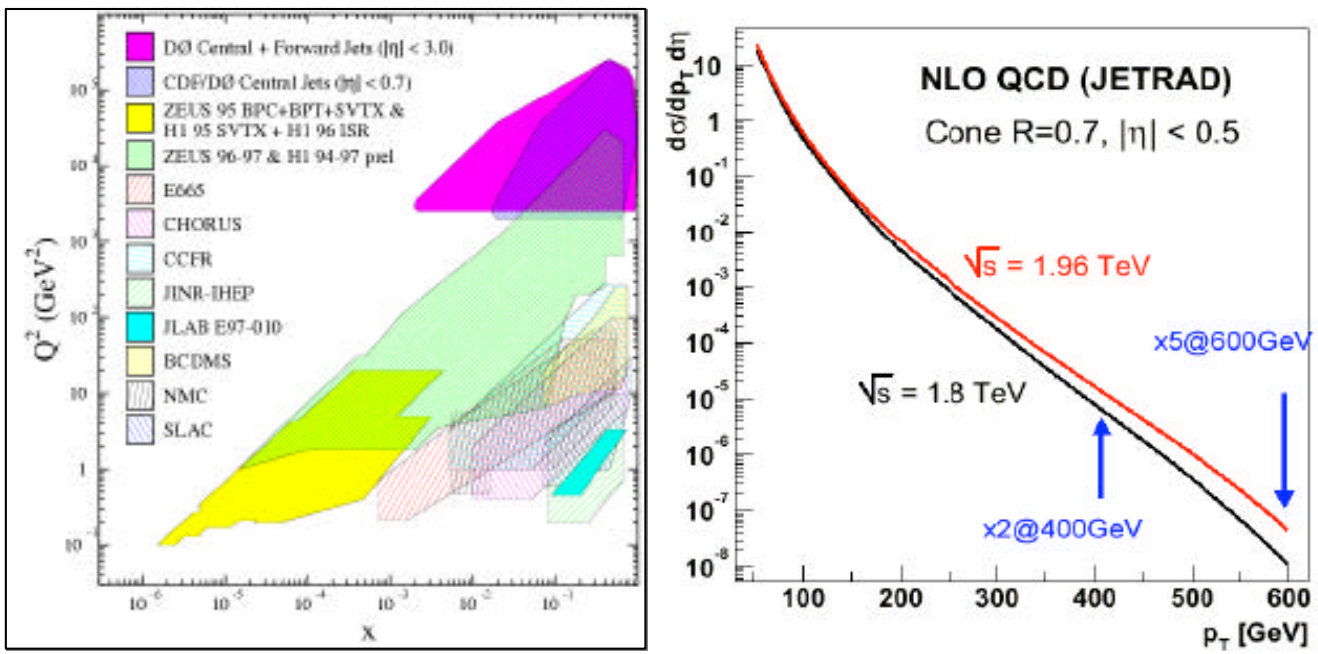

Figure 1: a) Structure function reach as a function of $x$ and $Q^{2}$ for a range of experiment, b) NLO prediction for the total jet cross section as a function of jet $p_{T}$ for center of mass energies corresponding to Run I and Run II.

\subsection{The DØ detector and data taking status}

Fermilab began proton antiproton collisions for RunII in March, 2001. Since that time, the luminosity has increased from an average peak luminosity of less than $10^{31} \mathrm{~cm}^{-2} \mathrm{sec}^{-1}$ to the current average peak luminosity of about $1.6 \times 10^{32} \mathrm{~cm}^{-2} \mathrm{sec}^{-1}$. In order to take advantage of the continuously increasing luminosity, $\mathrm{D} \emptyset$ installed a new tracking detector, and upgraded the trigger and data acquisition systems during a 3.5 month shutdown beginning mid-February 2006. The results reported here are from the data set taken before this date (Run IIa data). The delivered luminosity for Run IIa is $1.4 \mathrm{pb}^{-1}$ and the $\mathrm{D} \emptyset$ recorded luminosity from this period is $1.2 \mathrm{pb}^{-1}$. For details of the RunIIa detector see [1].

\section{$1.2 \quad$ Jets at DØ}

The jet algorithm has been improved for Run II to make the jets more infrared safe. The new jet algorithm, called the midpoint algorithm, defines a cone of radius $\boldsymbol{R}$ around all seed particles 
and adds particle four-vectors within the cone ("E-scheme") until a stable solution is found. In addition, to improve infrared safety, midpoints between seeds are also used as seeds. Finally all identical solutions are removed and $\mathrm{a}_{\mathrm{T}}{ }^{\mathrm{min}}$ cut is put on the jet. Jets with overlapping cones are split if the shared energy fraction $<50 \%$ and merged if the shared energy fraction is at least $50 \%$.

\subsubsection{Jet Energy Scale Calibration}

The main systematic contribution to the inclusive jet cross section analysis is the jet energy scale. In a $p \bar{p}$ collision, partons from the interaction hadronize into particles which in turn shower in the calorimeter. When reconstructing the jet, corrections are made to reconstruct the energy back to the particle level including corrections for pileup and electronic noise, average loss of energy in the calorimeter, and energy leaking into or out of the jet.

The jet energy scale is extracted from $\gamma+$ jet events. Jet $\boldsymbol{p}_{T}$ is balanced against the better measured $\boldsymbol{p}_{T}$ of the $\gamma$. The jet energy (particle level) is then measured using:

$$
E_{\text {jet }}^{\text {particle }}=\frac{E_{\text {jet }}^{\text {caloriment }}-E_{\text {offset }}}{R_{j e t} \bullet F_{S}}
$$

where $E_{\text {jet }}^{\text {particle }}$ is the jet energy at the particle level, $E_{\text {jet }}^{\text {calorimete }}$ is the jet energy as measured by the calorimeter, $E_{\text {offset }}$ is the energy not associated with the hard scatter (including underlying event), $R_{j e t}$ is the jet response which includes a response as a function of (uncorrected) jet energy and cone size and an additional term to correct for the detector $\eta$ dependence of the response, and $F_{s}$ is a correction for energy showering outside the jet cone and for neighboring jets showering into the jet cone.

The largest component to the scale correction is the absolute response, a correction of about $30 \%$. The uncertainty of the correction is a function of jet $\boldsymbol{p}_{T}$ and increases with increasing $\boldsymbol{p}_{T}$. Figure 3 shows the jet energy scale uncertainty at $\eta=0$. This preliminary jet energy scale was derived from a small subsample of $\gamma+$ jet events, $150 \mathrm{pb}^{-1}$, and the statistical uncertainty dominates especially at high jet $\boldsymbol{E}_{\boldsymbol{T}}$.

\subsubsection{Jet Physics}

High $\boldsymbol{p}_{T}$ jets are commonly thought of as hadronized partons from the hard parton scatter in the $p \bar{p}$ interaction. Of course this is an oversimplification of a complicated process that may also involve initial and/or final state hard and/or soft radiation, hadronization and/or fragmentation and underlying events from additional interactions. Some of these issues were discussed above in the context of the jet energy scale, and the jet energy scale correction will partially correct for these effects. Residual effects must be modeled in the Monte Carlo. Jet physics allows us to explore the models and make precision tests of QCD as well as look for new phenomena. In addition, insights gained at Tevatron energies will be useful estimators for LHC experiments. 


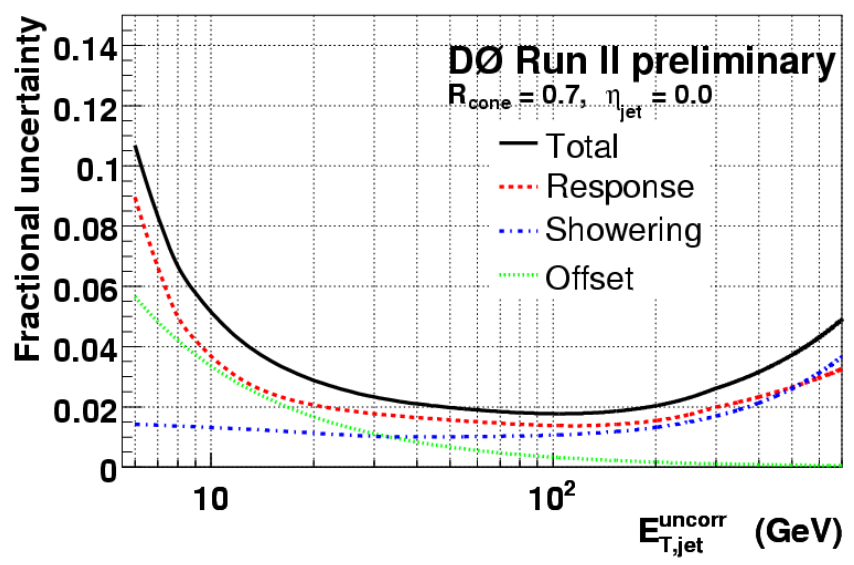

Figure 2: Jet Energy Scale uncertainty as a function of uncorrected jet $\mathrm{E}_{\mathrm{T}}$. Each correction is shown separately.

\subsubsection{Inclusive Jet Cross Section}

The inclusive jet cross section was measured based on $0.8 \mathrm{fb}^{-1}$ of data taken between 2002-2005, representing nearly all of the DØ Run IIa data [2] Events were triggered using single jet triggers based on energy deposited in calorimeter towers. The jets were reconstructed with the midpoint algorithm described above using a cone of $\mathrm{R}=0.7$.

After applying the jet energy scale and correcting for event and jet cut inefficiencies, the cross section was corrected for finite jet resolution. Because the cross section is a steeply falling function of $\boldsymbol{p}_{T}$, it is much more likely for jets of low $\boldsymbol{p}_{T}$ to migrate into a higher $\boldsymbol{p}_{T}$ bin than the other way around. This bias is corrected by using an unfolding technique using the measured jet $\boldsymbol{p}_{T}$ resolutions from dijet data. An Ansatz function is convoluted with the measured jet resolutions and fit iteratively to the $\boldsymbol{p}_{T}$ spectra. The data are then corrected by the ratio of the original to the folded Ansatz function. Another method used a folded version of Pythia and the measured jet $\boldsymbol{p}_{T}$ and rapidity $\boldsymbol{y}$ resolutions to cross check the result.

The resulting cross sections are compared with NLO pQCD in Figure 3a. The overall cross section has been normalized to theory at $\boldsymbol{p}_{T}=\mathbf{1 0 0} \mathrm{GeV}$ in the $|\boldsymbol{y}|<\mathbf{0 . 4}$ rapidity bin in order to remove the overall luminosity measurement uncertainty. In Figure $5 b$, the ratio of data to theory is plotted as a function of $\mathrm{p}_{\mathrm{T}}$. The yellow shaded region represents the total systematic uncertainty, which is dominated by the uncertainty on the jet energy scale. Dashed lines show the PDF uncertainties and the dot dashed lines show the theoretical prediction without threshold corrections. The rise of the data in the $|\boldsymbol{y}|<0.4$ and the fall in $0.4<|y|<0.8$ is due to the small statistics $\left(\sim 150 \mathrm{pb}^{-1}\right)$ of the data used to extract the preliminary jet energy scale. This preliminary measurement, however, is still able to constrain the PDFs.

Figure 4 shows a comparison of all central $D \emptyset$ inclusive jet results to date compared with pQCD with threshold and non-perturbative corrections. The comparison shows good agreement across a wide range of jet $\boldsymbol{p}_{T}$. 

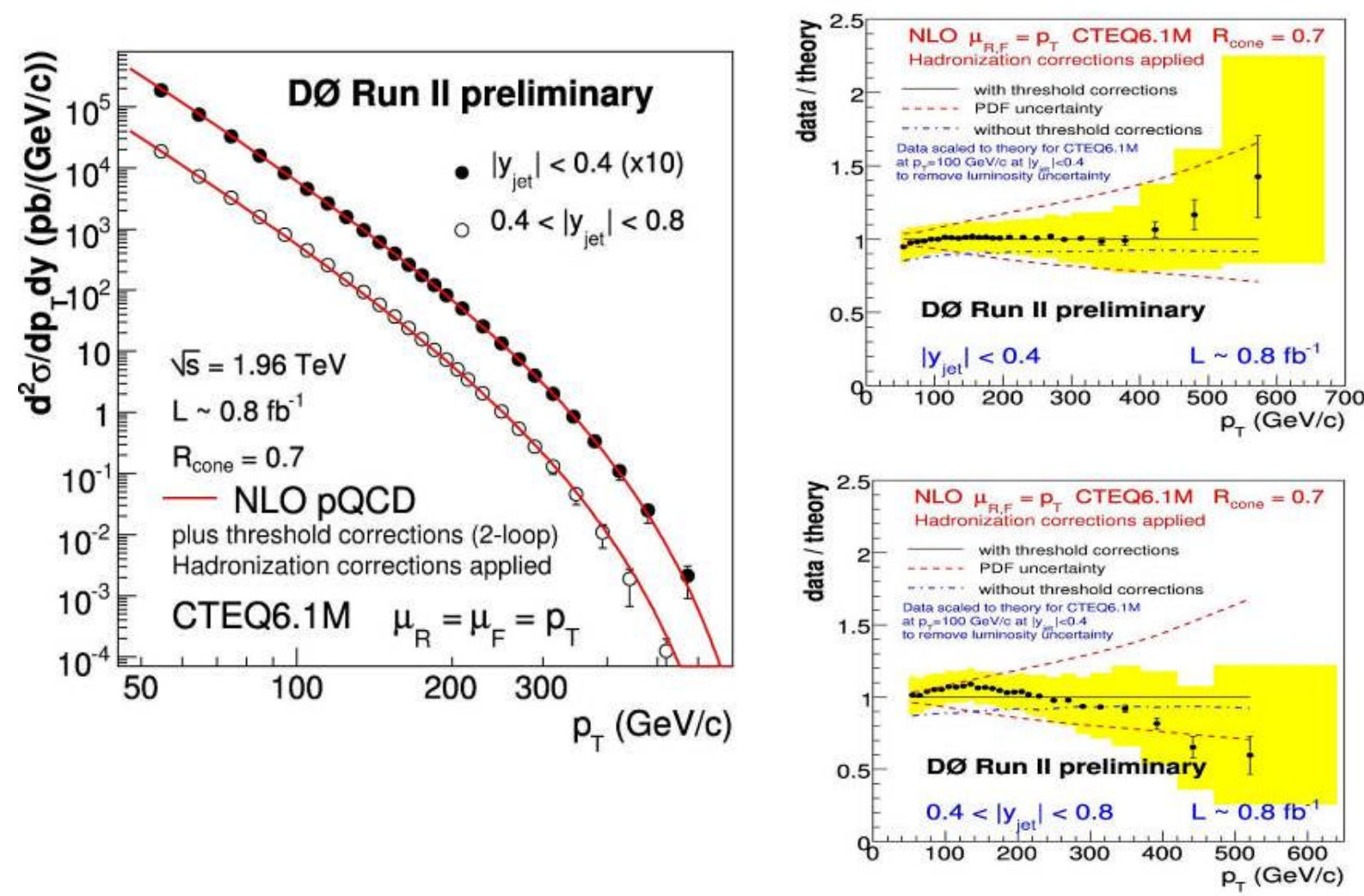

Figure 3: Inclusive Jet Cross Section a) and b)ratio with theory. The overall cross section is normalized to theory at $\boldsymbol{p}_{T}=100 \mathrm{GeV}$ in the $|\boldsymbol{y}|<0.4 \mathrm{bin}$.

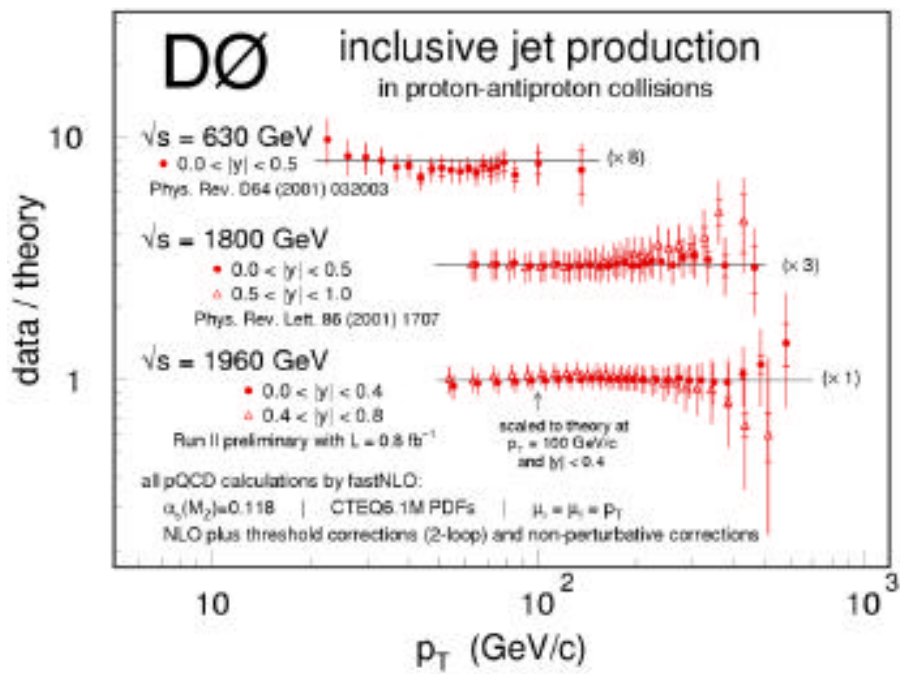

Figure 4: $D \varnothing$ inclusive jet cross section results, including this preliminary one. The comparison of data with NLO + corrections shows good agreement.

\subsubsection{Dijet Azimuthal Deccorelations}

While the inclusive jet cross section can constrain PDFs, the azimuthal angle between the leading and second leading jets in the inclusive jet system is a good probe of radiative 
processes in the event. In the absence of a hard or soft radiative process, the two leading jets are produced with equal and opposite $\boldsymbol{p}_{T}$ and thus completely correlated azimuthal angles $(\phi)$ with $\Delta \phi \sim \pi$. Initial or final state radiation disturbs this simple picture and leads to decorrelations in the jet azimuthal angle. Figure 5a) shows the measured jet $\Delta \phi$, made using $150 \mathrm{fb}^{-1}$ of the RunIIa data set [3]. The inner error bars represent the statistical uncertainty and the outer ones represent the quadratic sum of statistical and systematic uncertainty. In this measurement the dominant uncertainty is not the jet energy scale, but the jet resolution which can cause misidentification of the two eading jets, especially in multijet events. This has been corrected for in the data by using an unfolding technique similar to the one used for the inclusive jet cross section.
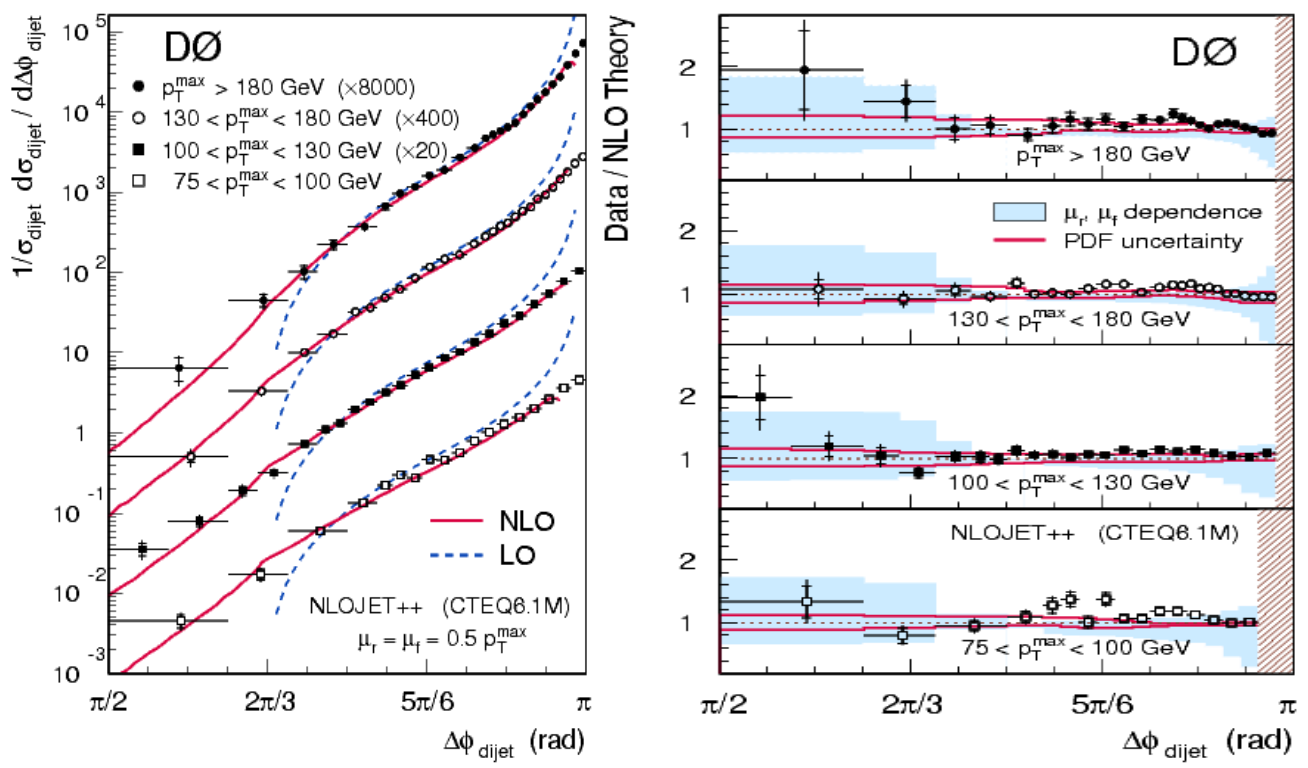

Figure 5: a) $\Delta \phi$ between the two leading jets in the inclusive jet event sample compared with LO and NLO predictions. b) Ratio of data over NLO as a function of $\Delta \phi$.

A salient feature of this measurement is the rapid rise at $\Delta \phi=\pi$ for back to back jets.

Events with smaller $\Delta \phi$ (decorrelated jets) are also present and are reasonably well described by the leading order Monte Carlo. However at $\Delta \phi=2 \pi / 3$, corresponding to three jet events, the leading order prediction does not follow the data. The next to leading order prediction does a much better job at both $\Delta \phi=\pi$ and smaller $\Delta \phi$. In Figure 5b) the measured $\Delta \phi$ is compared in ratio with the NLO prediction.

Figure 6a) shows the measured $\Delta \phi$ compared to Herwig and Pythia. The Herwig agreement is very good, but Pythia predicts too few small $\Delta \phi$ events. After tuning the parameter that controls the amount of initial state radiation, the agreement is very good over the full $\Delta \phi$ range. Figure 6b) shows a comparison of the measured $\Delta \phi$ to NLO, SHERPA and ALPGEN. Both SHERPA and ALPGEN agree well with the data. 

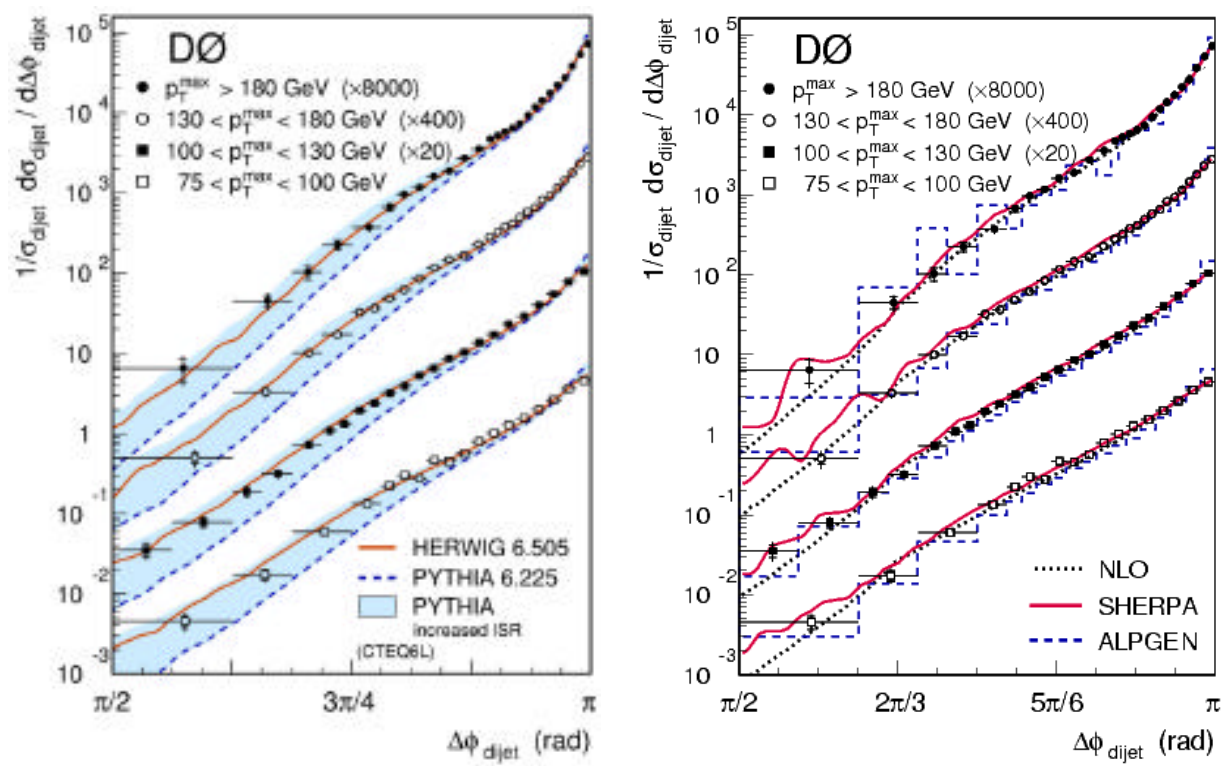

Figure 6: a) $\Delta \phi$ compared with Herwig and Pythia. b) $\Delta \phi$ compared with NLO, SHERPA and ALPGEN. See text for discussion.

\subsection{Isolated Photoproduction at DØ}

Photons in a hard scattering event can originate from either direct emission from the hard subprocess or during fragmentation. By requiring the photons to be isolated, the direct photon portion is enriched and the hard scattering itself is probed by the photon, which at lowest order is produced by $q \bar{q}$ annihilation or $q \gamma$ Compton scattering. Measuring photoproduction cross sections is a clean method of testing QCD, since the photon emerges unaltered from the hard subprocess without complications from jet algorithms and measurements.

The D $\varnothing$ analysis was based on $326 \mathrm{pb}^{-1}$ of Run IIa data [4]. Photons were reconstructed using clusters of energy in the calorimeter inside a cone of size $\boldsymbol{R}=\boldsymbol{0 . 2}$. In order to remove most of the hadronic jets, the fraction of energy deposited in the electromagnetic calorimeter was required to be greater than 0.9 . A veto on track(s) pointing to the cluster removed additional jet and most electron background. In addition the photon was required to be isolated by demanding that the energy in a cone of size $\boldsymbol{R}=\mathbf{0 . 4}$ around the photon contained less than $10 \%$ of the energy of the photon.

Because the jet cross section is several orders of magnitude larger than the photon cross section, there will still be some jets remaining in the sample with large electromagnetic fractions. In order to enhance the purity of the photon sample, a neural net was developed which used track and calorimeter variables within the photon cone. The neural net was trained on Pythia Monte Carlo (for the signal and for both QCD and electroweak backgrounds) and verified using Z->ee Data and Monte Carlo. The photon resolution is also measured in the data and is used to unfold the data, as in the inclusive jet measurement case. 

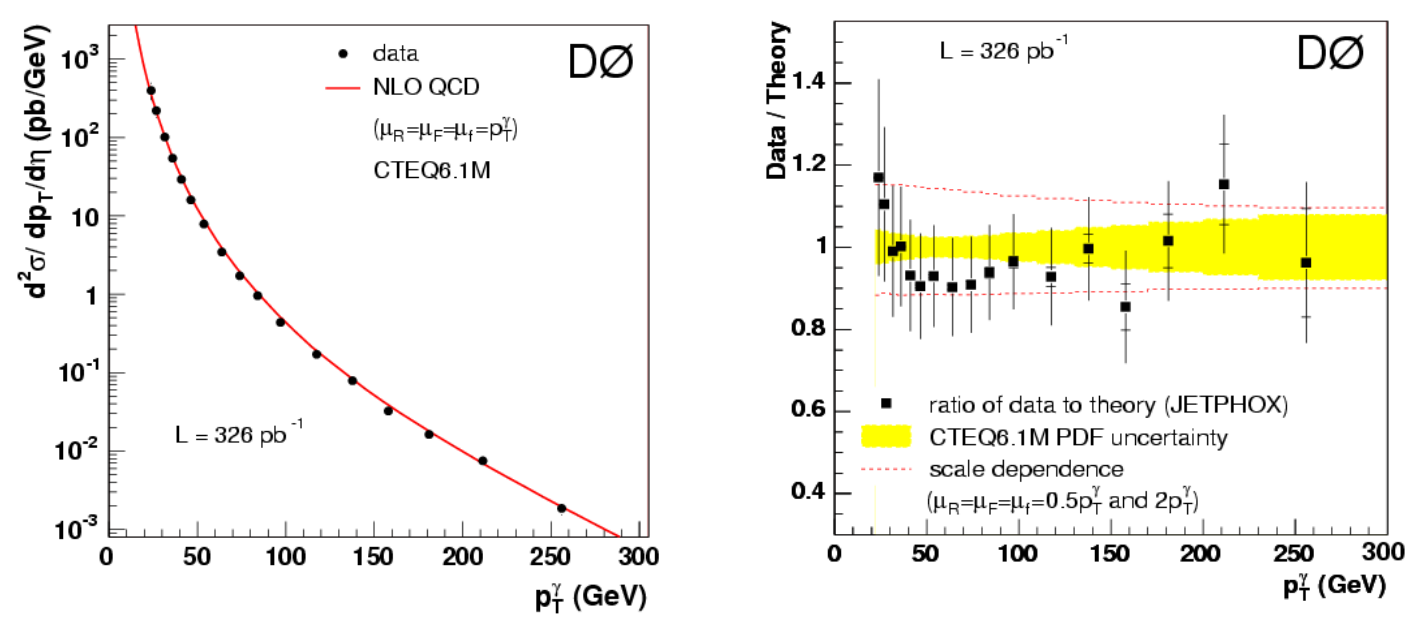

Figure 7: a) The inclusive isolated photon cross section for $|y|<0.9$ compared with NLO QCD and b) the inclusive isolated photon cross section ratio of data/theory.

Figure 7 shows the inclusive isolated photon cross section for $|y|<0.9$ together with the NLO QCD calculations from JETPHOX/Vogelsang using CTEQ6.1M PDFs. The central value of the prediction changes by less than 7\% when the PDFs are replaced by MRST2004 or Alekhin2004 PDFs. The agreement is good, however both experimental and theoretical uncertainties need to be reduced in order to have sensitivity to PDFs. The experimental uncertainty is dominated by the photon purity and theoretical uncertainties could be improved with resummation and NNLO calculations.

\subsection{Elastic and Diffractive Scattering at Do}

About $40 \%$ of the total $p \bar{p}$ cross section comes from elastic and diffractive scattering. In elastic scattering the protons are scattered through an extremely small angle with no energy loss and hence no additional particles produced. In diffractive scattering there are three possibilities. In the single diffraction process one of the protons remains intact and the other dissociates, in the diffractive central production case both protons remain intact with small energy loss and there are additional particles in the central rapidity region. Finally in the double diffraction case, both protons dissociate.

\subsubsection{Detector Overview}

While the topology discussed above for elastic and diffractive events seems clean, in practice there are underlying events and detector noise effects that muddy the waters. In order to be able to unambiguously reconstruct the proton and antiproton in the elastic and diffractive cases, DØ has installed a Forward Proton Detector (FPD), depicted in Figure 8. 


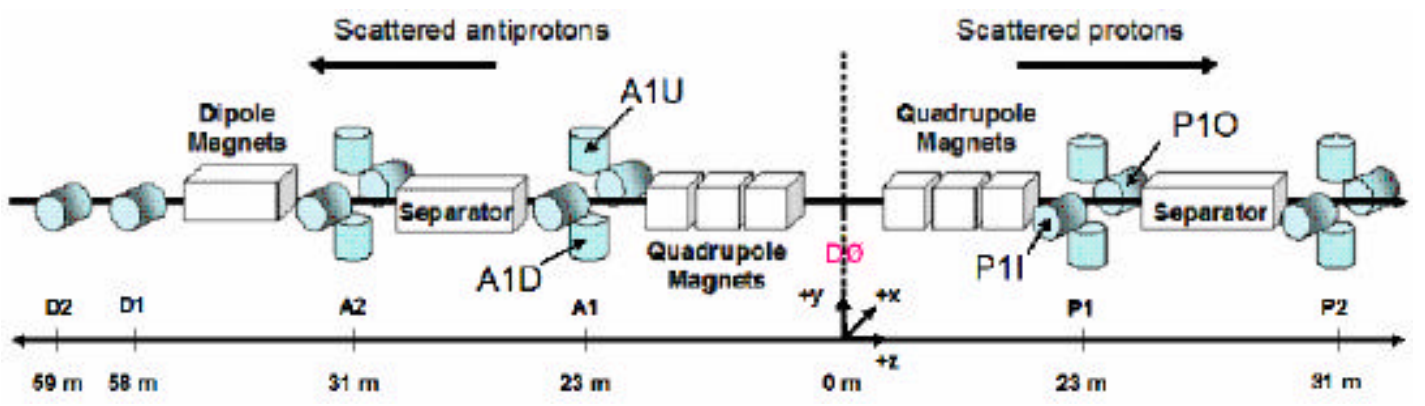

Figure 8 The Forward Proton Detector spectrometer. See the text for more description.

The FPD detector is comprised of 18 Roman Pots in 9 spectrometers arranged in 6 stations called castles. In each of the quadrupole stations, there are 4 Roman Pots, two in the vertical (up, down) and two in the horizontal (in, out) positions. Each Roman Pot houses a detector made up of three planes $(\boldsymbol{U}, \boldsymbol{X}, \boldsymbol{V})$ of scintillating fibers of width $800 ? \mathrm{~m}$. Each plane is formed of two layers of parallel fibers $\left(U-U^{\prime}, X-X^{\prime}, V-V^{\prime}\right)$ with the primed layers shifted by a third of a fiber width. The active area of the scintillating detectors are $17.5 \times 17.5 \mathrm{~mm}^{2}$. On the $\bar{p}$ end of the spectrometer there are two additional stations with one Roman Pot each. A dedicated trigger has been designed and implemented to provide a fast response to protons and antiprotons passing through the detectors. The triggers have been used to collect large samples of elastic and diffractive scattered events

Reconstructed hits are used to measure the fractional momentum loss ? and the four momentum transfer squared $t$ of the scattered beam proton and antiprotons. The FPD covers the region $t_{\min }<|t|<4.5 \mathrm{GeV}^{2}$, where $t_{\min }$ is determined by how close the detectors can be brought to the beam.

\subsubsection{Status of Data Taking and Analysis}

From January 2004 onward, the FPD was an integrated part of DØ running. The detector has been successfully calibrated and aligned. Figure 9 shows the correlation in $\boldsymbol{x}$ (right) and $\boldsymbol{y}$ (left) between the two dipole detectors. The data are a mix of beam halo and diffractively or elastically scattered protons. Two bands are evident in the $\boldsymbol{y}$ coordinate, corresponding to the bend plane of the dipole magnet. The diagonal band comes from particles at beam energy (halo) and the off diagonal band is caused by particles with energy slightly less than beam energy (the scattered antiproton).

\subsection{Conclusion and Outlook}

$\mathrm{D} \emptyset$ has measured the inclusive jet cross section using $0.8 \mathrm{fb}^{-1}$ of data in two rapidity bins in the central region $|y|<0.8$. The jet energy scale contributes the largest uncertainty to the measurement, which is nevertheless precise enough to be sensitive to PDFs. A future measurement using a more precise jet energy scale and including higher rapidity bins is in the works and will be out soon.

The diffractive spectrometer has been aligned and calibrated and signals have been recorded in all nine spectrometers. During normal physics runs the spectrometer probes $|t|$ down 
to about $0.8 \mathrm{GeV}^{2}$, while special runs at low luminosity and high $\beta *$ allowed the Roman Pots to be inserted closer to the beam, thus probing $|t|<0.2 \mathrm{GeV}^{2}$. Analysis of this data is in progress.
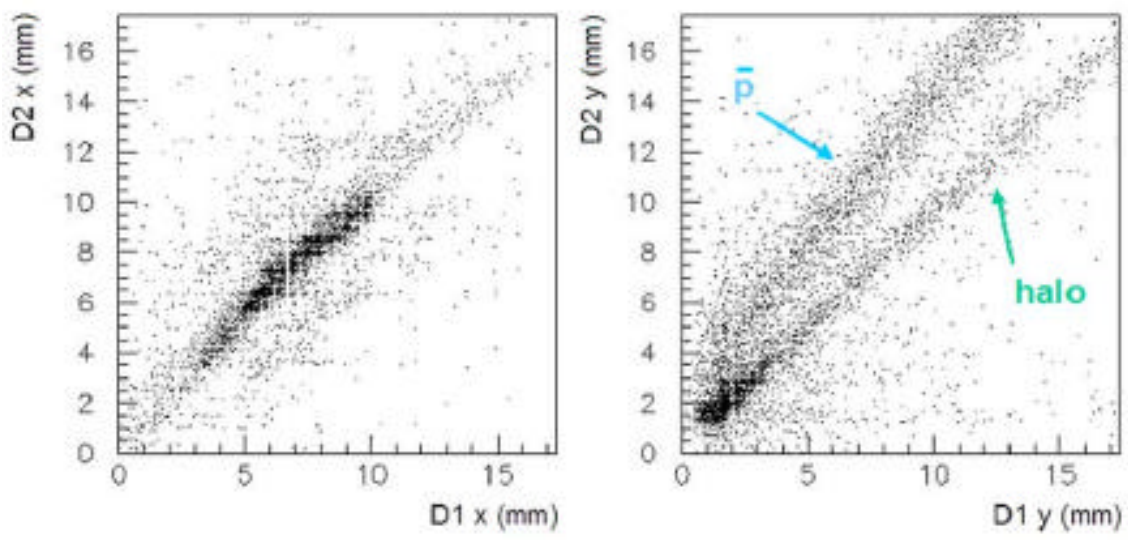

Figure 9: Correlation in $\boldsymbol{x}$ (right) and $\boldsymbol{y}$ (left) between the two dipole detectors of the FPD. A clear separation is seen between beam halo and the scattered antiproton.

\section{References}

[1] V.M. Abazov et al. (the DØ Collaboration), The Upgraded DØ Detector, Nuclear Instruments and Methods, A565, 463-537 (2005).

[2] See M. Voutilainen, Proceedings of the XIV International Workshop on Deep Inelastic Scattering (2006) and references therein.

[3] V.M. Abazov et al. (the DØ Collaboration), Measurement of Dijet Azimuthal Decorrelations at Central Rapidities in $p \bar{p}$ Collisions at $\sqrt{s}=1.96 \mathrm{TeV}$, Physics Review Letters 94, 221801 (2005) and references therein.

[4] V.M. Abazov et al. (the DØ Collaboration), Measurement of the Isolated Photon Cross Section in $p \bar{p}$ Collisions at $\sqrt{s}=1.96 \mathrm{TeV}$, Physics Letters B639, 151 (2006) and references therein. 